\title{
Synthesis of L-proline derived cyclic $\beta$-turn mimics via ring closing metathesis
}

\author{
Rajesh B. M, Madhushree Bhattacharya, Biswadip Banerji, and Javed Iqbal* \\ Discovery Research, Dr. Reddy's Laboratories Ltd, \\ Bollaram Road, Miyapur, Hyderabad 500 049, India \\ E-mail: javediqbaldrf@hotmail.com
}

\section{Dedicated to Professor P. T Narasimhan on the occasion of his $75^{\text {th }}$ anniversary}

(received 04 May 04; accepted 28 Dec 04; published on the web 16 Jan 05)

\begin{abstract}
Acyclic peptides having pent-4-enoyl at $N$-terminal and $O$-allyl at $C$-terminal as linkers can be transformed to $\beta$-turn mimics via ring closing metathesis using Grubb's catalyst. The chirality of Phenylalanine residue controls the cyclization during RCM, which leads to the concomitant formation of 6-hydroxy-caproic acid as a linker during cyclization.
\end{abstract}

Keywords: $\beta$-Turn, ring closing metathesis, nOe spectrum

\section{Introduction}

The use of peptidomimetics to overcome the limitations inherent in the physical characteristics of peptides has become an important strategy for improving the therapeutic potential of peptides. Generally, peptidomimetics are derived from a lead peptide sequence where structural modifications have been incorporated to improve binding affinity and/or metabolic resilience. Although many peptide sequences have been identified as potent bioactive agents, there are fundamental limitations associated with the development of peptides as therapeutics. The inherent conformational flexibility of a small peptide results in a myriad of conformations adopted by the peptide. In addition, there are a number of metabolic limitations restricting the use of peptides as therapeutics. Poor permeability across membranes, proteolytic degradation, rapid clearance and in some cases poor solubility and a tendency to aggregate, all contribute to low oral availability of peptide based therapeutics ${ }^{1}$.

Peptidomimetics based on $\beta$-turns ${ }^{2,3}$ are attractive mimics of the 'bioactive conformations' because numerous peptides elicit a biological response via such a conformation. In an ongoing program in our laboratory on the discovery of small molecule ligands inhibiting HIV-I protease, we have undertaken the synthesis of cyclic tripeptides derived from Gly-Pro-XAA and Phe-ProYAA residues. In this process we are trying to understand the role of preceding and succeeding 
amino acids to proline during RCM. The choice of these tripeptides is dictated by the fact that HIV protease is very specific in cleaving the peptide bond between later two residues and the synthesis of the cyclic $\beta$-turn mimics derived especially from Phe-Pro may lead to the development of small inhibitors characterized by high affinity, bioavailability and resistance to proteolytic degradation. In this paper, we report the synthesis of cyclic peptides by ring closing metathesis (RCM) reaction.

\section{Results and Discussion}

In order to probe the conformational features of such cyclizations, it is envisioned that tripeptides from $X A A-P r o-Y A A$ would provide an interesting study on their folding properties. We have started with 'gly-pro-gly' motif and synthesized the tripeptide $15(\mathrm{R} 1=\mathrm{R} 2=\mathrm{R} 3=\mathrm{R} 4=\mathrm{H})$ as shown in Scheme1.

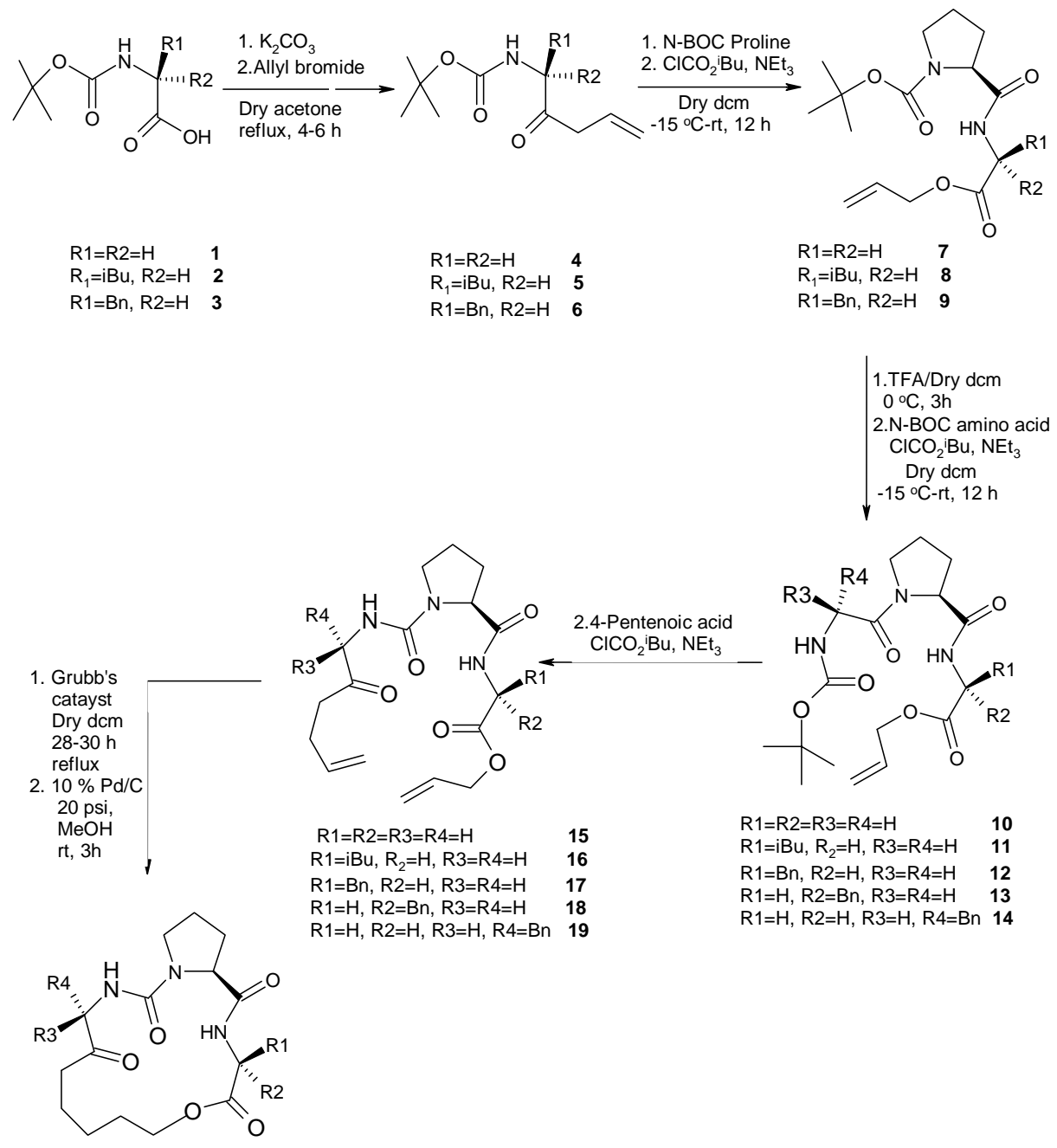

$\mathrm{R} 1=\mathrm{iBu}, \mathrm{R}_{2}=\mathrm{H}, \mathrm{R} 3=\mathrm{R} 4=\mathrm{H} \quad 16 \mathrm{C}$ $\mathrm{R} 1=\mathrm{Bn}, \mathrm{R} 2=\mathrm{H}, \mathrm{R} 3=\mathrm{R} 4=\mathrm{H} \quad 17 \mathrm{C}$ $\mathrm{R} 1=\mathrm{H}, \mathrm{R} 2=\mathrm{H}, \mathrm{R} 3=\mathrm{H}, \mathrm{R} 4=\mathrm{Bn} 19 \mathrm{C}$

\section{Scheme 1}


It is generally known that Pro-Gly residues in proteins are turn inducers and introduction of a glycine residue at both ends of L-proline should add an additional element of turn inducing property, which may help in $\beta$-turn formation. We have used $N$-pentenoyl and $O$-allylester at the two ends of these peptides so that, RCM cyclization leads to the formation of cyclic peptides containing 6-hydroxy-4, 5-dehydro-caproic acid as a linker.

The solvent titration study of $\mathbf{1 5}$ showed the absence of any intramolecular H-bonding \& preorganized secondary structure. The acyclic peptide 15 when subjected to RCM using Grubb's catalyst, failed to cyclize and this, was attributed to the flexible conformation of the molecule. It is known that glycine residue in particular is free from $\varphi$ and $\psi$ angle bias compared to the other substituted amino acids and that it is capable of imparting a large degree of rotational freedom in the molecule. Consequently, the two termini of the peptide backbone can go far apart from each other eluding the possibility of RCM cyclization.

Considering 'glycine' unit as the main cause for imparting this flexibility into the molecule we decided to use chiral amino acid like phenylalanine in place of the former at C-terminus of proline. Eventually, we synthesized tripeptide 17 (scheme-1). In order to prove the presence of $\beta$ turn we carried out a deuterium exchange study on 17 using $\mathrm{CD}_{3} \mathrm{OD}$ in $\mathrm{CDCl}_{3}$ which showed that no deuterium exchange took place for the bonded amide proton (Phe $\mathrm{NH} \delta=7.4 \mathrm{ppm}$, IR $=$ $3264 \mathrm{~cm}^{-1}$ ) even after 5 hours. Having established the presence of the $\beta$-turn in 17, we subjected it to RCM using Grubb's ruthenium catalyst (20 mol \%). It was gratifying to find that the acyclic peptide 17 cyclized smoothly to the corresponding cyclic peptide, which was hydrogenated to obtain the cyclic peptide 17C (scheme-1) in good yield. It is also interesting to note that the $\beta$ turn, which was present in the acyclic precursor 17, was retained in the cyclic peptide 17C (Phe $\mathrm{NH}, \delta=7.45 \mathrm{ppm}, \mathrm{IR}=3314 \mathrm{~cm}^{-1}$ ). The presence of $\beta$-turn was also confirmed by nOe studies using non-quantitative NOESY type spectra (Figure1).

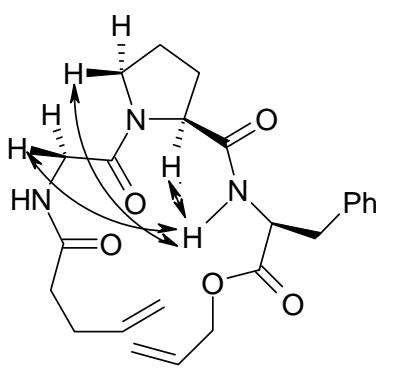

17

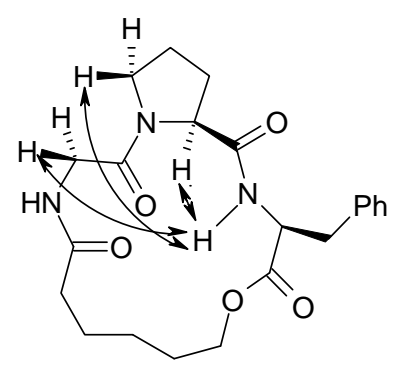

17C

Figure 1

Based on our earlier work ${ }^{4}$ it is interesting to note that, the cyclic peptide (20) derived by cyclization of pent-4-enoyl-Gly-L-Pro-L-Phe- $N$-allylamide using Grubb's catalyst (20 mol \%) is organized via a $31_{10}$-helix (Phe NH and allylic NH was H-bonded) where the donor and acceptor partners differ from the corresponding cyclic peptide 17C (Phe-NH is H-bonded) (Figure 2). 

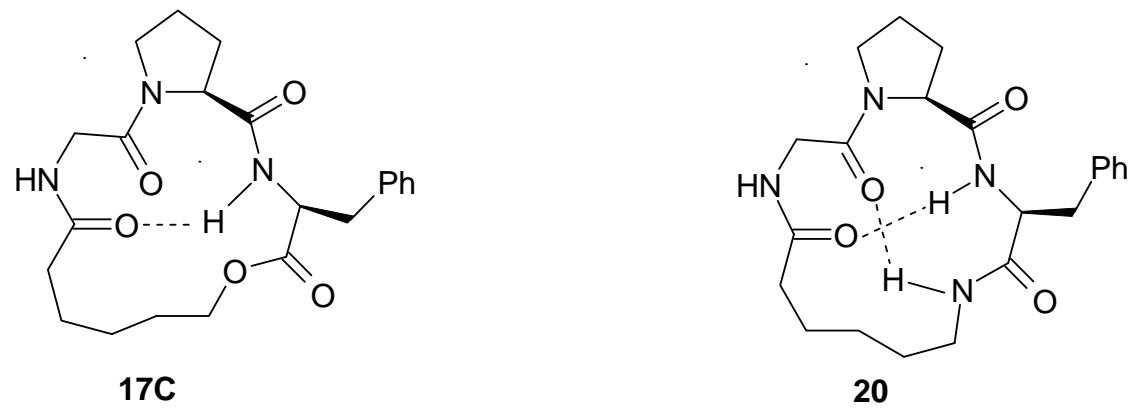

Figure 2

We were also interested in investigating the role of chirality in this peptide and thus synthesized the corresponding acyclic peptide 18 (scheme-1) containing D-phenylalanine instead of L-phenylalanine in 17. Although the solution NMR studies confirmed the presence of a 10memebered $\beta$-turn in 18 (Leu NH $=6.97, \mathrm{IR}=3308 \mathrm{~cm}^{-1}$ ), RCM using Grubb's catalyst ( $30 \mathrm{~mol} \%$ ) failed to give the cyclic peptide. Failure of $\mathbf{1 8}$ to undergo RCM reaction may be attributed to the importance of chirality ( $\varphi$ and $\psi$ angle) of amino acid at $\mathrm{C}$-terminal of proline.

Based on the result obtained above we wanted to probe the influence of the side chain amino acid residue at C-terminus of proline and accordingly synthesized acyclic tripeptide $\mathbf{1 6}$ (scheme1). Interestingly the solution NMR study of the peptide $\mathbf{1 6}$ indicated the participation of leucine $\mathrm{NH}$ in intramolecular H-bonding (Leu NH, $\delta=6.94 \mathrm{ppm}, \mathrm{IR}=3321 \mathrm{~cm}^{-1}$ ), suggesting that here the two terminal olefinic bonds may come in close proximity. Indeed, it was found that corresponding cyclic peptide 16C was obtained when 16 was subjected to RCM (20 mol \% Grubb's catalyst) and hydrogenation conditions (Leu NH, $\delta=7.2 \mathrm{ppm}, \mathrm{IR}=3315 \mathrm{~cm}^{-1}$ ). The presence of $\beta$-turn in $\mathbf{1 6}$ and $\mathbf{1 6 C}$ was also confirmed by nOe studies using non-quantitative NOESY type spectra (Figure 3).

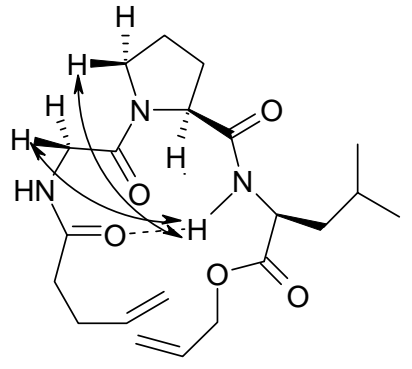

16

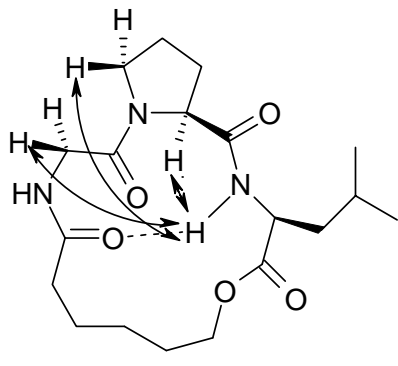

16C

Figure 3

Having established the requirement of L-amino acid at C-terminal of proline in $\mathbf{1 6}$ and $\mathbf{1 7}$ to undergo RCM reactions, we wanted to probe the role of chiral amino acid at N-terminus of proline and accordingly synthesized pent-4-enoyl-D-Phe-Pro- $O$-allylester 19 according to the synthetic procedure outlined in scheme-1. The intramolecular hydrogen bonding properties of 19 was studied by its solution NMR, which showed the Glycine NH to be intramoleculary hydrogen 
bonded (Gly NH 7.40 ppm, IR $=3282 \mathrm{~cm}^{-1}$ ) in a 10-membered $\beta$-turn fashion. Since this acyclic peptide is pre-organized with a intramoleular hydrogen bonding, we expected this peptide to undergo smooth cylization and indeed, we found that 19 undergoes ring closing metathesis reaction smoothly giving the unsaturated cyclic product which was hydrogenated to give 19C (Gly NH 7.58 ppm, IR $=3395 \mathrm{~cm}^{-1}$ ) The nOe of acyclic and cyclic peptides are shown in (Figure 4).

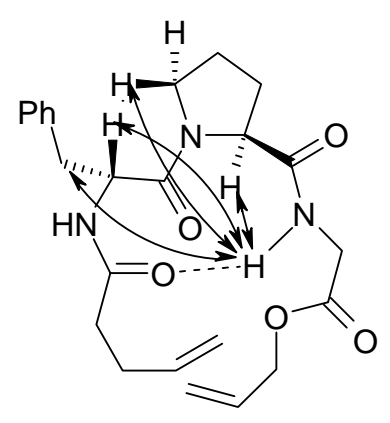

19

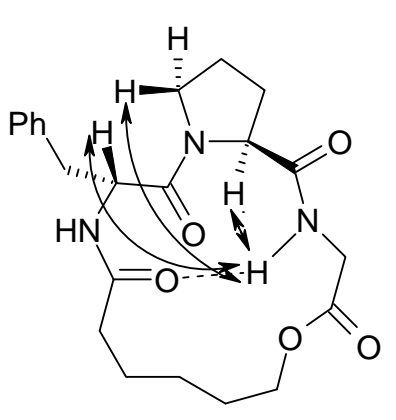

19C

\section{Figure 4}

\section{Conclusions}

We have synthesized cyclic peptide $\beta$-turn mimics and the studies described above indicate that the role of chirality of phenylalanine dictates the RCM cyclization when proline is present at $i+2$ position. Therefore RCM reactions act as a probe for the $\beta$-turn conformation in small peptides and these small molecules mimetic can be used as a tool to understand the Protein-Ligand interactions. We are further pursuing our studies to understand the role of L-amino acids preceding L-Proline for synthesizing cyclic peptides by RCM.

\section{Experimental Section}

General Procedures. Melting points were determined manually. ${ }^{1} \mathrm{H}$ NMR spectra were recorded on Varian Mercury $400 \mathrm{MHz}$ and Varian Gemini-200 $\mathrm{MHz}$ spectrometer in $\mathrm{CDCl}_{3}$ with TMS as internal standard. Chemical shifts are quoted in ppm and $J$ values are given in Hertz. IR spectra were recorded on an FT-IR spectrophotometer from the Perkin-Elmer 1600 series. Mass spectra were recorded on a Hewlett Packard 5989A LC-mass spectrometer using isobutene as chemical ionizer gas. Optical Rotation was recorded using JASCO DIP 370 spectrophotometer.

\section{General procedure for peptide coupling}

To a stirred solution of pent-4-enoyl-XAA acid in DCM at $0{ }^{\circ} \mathrm{C}$ was added triethylamine (1equiv), followed by isobutyl chloroformate (1 equiv) and the mixture was stirred for 5 min and then finally this was added to XAA-allylester followed by triethylamine (1 equiv). The reaction 
mixture was stirred for $5 \mathrm{~h}$ at room temperature and dilute with dichloromethane. The organic layer was washed with 5\% sodium bicarbonate solution, cold saturated citric acid solution and finally with brine. Drying over sodium sulfate and evaporating the solvent under vacuum yielded the crude compound which was chromatographed (100-200 silica gel, $\left.\mathrm{MeOH}: \mathrm{CHCl}_{3}\right)$ to afford the desired peptide in good yields.

\section{General procedure for RCM}

To a stirred solution of Grubb's ruthenium catalyst (10 mol\%) in dry dichloromethane (in high dilution) under nitrogen was added acylic peptide dissolved in dry dichloromethane slowly over a period of $30 \mathrm{~min}$ and the mixture refluxed for $12 \mathrm{~h}$ after which a further portion of catalyst (10 mol\%) was added to the reaction mixture and refluxing continued. Finally, after $28-30 \mathrm{~h}$ the reaction was exposed to air and the solvent was evaporated to afford the crude product, which was chromatographed (100-200 silica gel, $\left.\mathrm{MeOH}: \mathrm{CHCl}_{3}\right)$ to afford the unsaturated cyclic peptide as a mixture of $E$ and $Z$ isomers in 50-60\% yield.

\section{General procedure for hydrogenation}

To a stirred suspension of $10 \% \mathrm{Pd} / \mathrm{C}(15 \mathrm{mg})$ in methanol $(2 \mathrm{ml})$ was added a solution of unsaturated cyclic peptide $(50 \mathrm{mg})$ in $5 \mathrm{ml}$ of $\mathrm{MeOH}$ at room temperature. The reaction flask was evacuated and hydrogenated using a $\mathrm{H}_{2}$ gas balloon (approx-20 psi) for $3 \mathrm{~h}$. The catalyst was filtered off using a celite pad and the filtrate was concentrated to afford the crude compound which was purified using (100-200 silica gel, $\left.\mathrm{MeOH}: \mathrm{CHCl}_{3}\right)$ to afford the cyclic peptide in quantitative yields.

\section{Compound characterization}

Spectral data for 4. $\mathrm{R}$ (Neat): $3382,2928,1715,1166 \mathrm{~cm}^{-1},{ }^{1} \mathrm{H} \mathrm{NMR}\left(400 \mathrm{MHz}, \mathrm{CDCl}_{3}\right): \delta 5.9-$ $5.8(\mathrm{~m}, 1 \mathrm{H}), 5.36-5.31(\mathrm{~m}, 1 \mathrm{H}), 5.2-5.2(\mathrm{~m}, 1 \mathrm{H}), 5.00(\mathrm{bs}, \mathrm{NH}), 4.6-4.6(\mathrm{~m}, 2 \mathrm{H}), 3.9-3.9(\mathrm{~m}, 2 \mathrm{H})$, $1.46(\mathrm{~s}, 9 \mathrm{H})$, Mass (CI method) $(\mathrm{m} / \mathrm{z}): 216\left(\mathrm{M}^{+}+1,100\right)$,

Spectral data for $5 .[\alpha]_{\mathrm{D}}=-35.8(\mathrm{C}, 1.0, \mathrm{MeOH})$, IR (Neat): 3374, 2927, 1717, $1166 \mathrm{~cm}^{-1},{ }^{1} \mathrm{H}$ NMR $\left(400 \mathrm{~Hz}, \mathrm{CDCl}_{3}\right): \delta$ 5.9-5.8 (m, 1H), 5.35-5.23 (m, 2H), $4.8(\mathrm{bs}, \mathrm{NH}), 4.63-4.57(\mathrm{~m}, 2 \mathrm{H})$, 4.3-4.2(bs, $1 \mathrm{H}), 1.7-1.6(\mathrm{~m}, 2 \mathrm{H}), 1.5-1.47(\mathrm{~m}, 1 \mathrm{H}), 1.46(\mathrm{~s}, 9 \mathrm{H}), 0.96-0.93(\mathrm{~m}, 6 \mathrm{H})$, Mass (CI method) (m/z): $272\left(\mathrm{M}^{+}+1,\right), 216(100)$.

Spectral data for 6. $[\alpha]_{\mathrm{D}}=-10.80(\mathrm{C}, 0.5, \mathrm{MeOH})$, IR (Neat): 3380, 2929, 1717, $1167 \mathrm{~cm}^{-1},{ }^{1} \mathrm{H}$ NMR (400 MHz, $\left.\mathrm{CDCl}_{3}\right): \delta$ 7.30-7.21 (m, 4H), 7.14-7.12 (m, 2H), 5.9-5.8 (m, 1H), 5.35-5.23 $(\mathrm{m}, 2 \mathrm{H}), 4.8(\mathrm{bs}, 1 \mathrm{H}), 4.63-4.57(\mathrm{~m}, 2 \mathrm{H}), 3.14-3.03(\mathrm{~m}, 2 \mathrm{H}), 1.41(\mathrm{~s}, 9 \mathrm{H})$, Mass (CI method) $(\mathrm{m} / \mathrm{z}): 306\left(\mathrm{M}^{+}+1\right), 250(100)$.

Spectral data for 7. IR (Neat): 3321, 2977, 1754, $1698 \mathrm{~cm}^{-1},{ }^{1} \mathrm{H}$ NMR (400 MHz, $\left.\mathrm{CDCl}_{3}\right): \delta$ 5.95-5.85 (m, 1H), 5.35-5.24 (m, 2H), $4.64(\mathrm{~d}, \mathrm{~J}=5.9 \mathrm{~Hz}, 2 \mathrm{H}), 4.32(\mathrm{~d}, J=5.9 \mathrm{~Hz}, 2 \mathrm{H}), 4.14-$ $3.98(\mathrm{~m}, 3 \mathrm{H}), 3.98(\mathrm{bs}, \mathrm{NH}), 2.16-2.15(\mathrm{~m}, 1 \mathrm{H}), 1.92-1.85(\mathrm{~m}, 3 \mathrm{H}), 1.47(\mathrm{~s}, 9 \mathrm{H})$, Mass $(\mathrm{CI}$ method) (m/z): $313\left(\mathrm{M}^{+}+1,10\right), 257$ (35), 213 (100) 
Spectral data for 8. $[\alpha]_{\mathrm{D}}=-82.8(\mathrm{C}, 0.5, \mathrm{MeOH})$, IR (Neat): 3316, 2960, 1744, $1698 \mathrm{~cm}^{-1},{ }^{1} \mathrm{H}$ NMR (400 MHz, $\left.\mathrm{CDCl}_{3}\right): \delta 6.4(\mathrm{bs}, \mathrm{NH}), 5.95-5.85(\mathrm{~m}, 1 \mathrm{H}), 5.34-5.23(\mathrm{~m}, 2 \mathrm{H}), 4.61(\mathrm{~d}, J=$ $5.9 \mathrm{~Hz}, 2 \mathrm{H}), 4.29(\mathrm{bs}, 2 \mathrm{H}), 3.42-3.40$ (m, 2H), 2.167 (bs, 1H), 1.87 (bs, 3H), 1.66-1.61 (m, 3H), $1.46(\mathrm{~m}, 9 \mathrm{H}) 0.92-0.91(\mathrm{~m}, 6 \mathrm{H})$, Mass (CI method) (m/z): $370\left(\mathrm{M}^{+}+2,50\right), 314(64), 270(100)$

Spectral data for 9. $[\alpha]_{\mathrm{D}}=-55.4(\mathrm{C}, 0.5, \mathrm{MeOH})$, IR (Neat): 3319, 2976, 1744, $1697 \mathrm{~cm}^{-1},{ }^{1} \mathrm{H}$ NMR (400 MHz, $\left.\mathrm{CDCl}_{3}\right): \delta$ 7.28-7.20 (m, 3H), 7.11-7,10 (m, 2H), $6.46(\mathrm{bs}, \mathrm{NH}), 5.91-5.82(\mathrm{~m}$, $1 \mathrm{H}), 5.32-5.23(\mathrm{~m}, 2 \mathrm{H}), 4.87(\mathrm{bs}, 1 \mathrm{H}), 4.60(\mathrm{~d}, J=5.1 \mathrm{~Hz}, 2 \mathrm{H}), 4.22(\mathrm{bs}, 1 \mathrm{H}), 3.37-3.29(\mathrm{~m}, 2 \mathrm{H})$, $3.19(\mathrm{dd}, J=14 \mathrm{~Hz}$ and $J=6 \mathrm{~Hz}, 1 \mathrm{H}), 3.02(\mathrm{dd}, J=13.6 \mathrm{~Hz}$ and $J=6.8 \mathrm{~Hz}, 1 \mathrm{H}), 2.25-2.20(\mathrm{~m}$, $1 \mathrm{H}), 1.87-1.83(\mathrm{~m}, 3 \mathrm{H}), 1.42(\mathrm{~s}, 9 \mathrm{H})$, Mass (CI method) $(\mathrm{m} / \mathrm{z}): 404\left(\mathrm{M}^{+}+2,16\right), 347(26), 304$ (100)

Spectral data for 10. IR (Neat): $3325,2978,1749,1654 \mathrm{~cm}^{-1},{ }^{1} \mathrm{H}$ NMR (400 MHz, $\left.\mathrm{CDCl}_{3}\right): \delta$ 7.30 (bs, NH), 5.95-5.85 (m, 1H), $5.40(\mathrm{bs}, \mathrm{NH}), 5.35-5.24(\mathrm{~m}, 2 \mathrm{H}), 4.64(\mathrm{~d}, J=8.4 \mathrm{~Hz}, 2 \mathrm{H})$, 4.08-4.06 (m, 1H), 4.04-4.01 (m, 2H), 3.97-3.87 (m, 2H), 3.58-3.52 (m, 1H), 3.42-3.37 (m, 1H), 2.44-2.41 ( m, 1H), 2.18-2.10 ( m, 1H), 2.08-2.00 (m, 1H), 1,91-1.84 ( m,1H), 1.44 (s, 9H), Mass (CI method) (m/z): $371\left(\mathrm{M}^{+}+2,41\right), 314(61), 270(100)$.

Spectral data for 11. $[\alpha]_{D}=-108.00(\mathrm{C}, 0.5, \mathrm{MeOH})$, IR (Neat): 3321, 2960, 2873, 1742, 1717 , $1654 \mathrm{~cm}^{-1},{ }^{1} \mathrm{H}$ NMR $\left(400 \mathrm{MHz}, \mathrm{CDCl}_{3}\right): \delta 7.06(\mathrm{~d}, J=7.2 \mathrm{~Hz}, \mathrm{NH}), 5.95-5.85(\mathrm{~m}, 1 \mathrm{H}), 5.40(\mathrm{bs}$, $1 \mathrm{H}), 5.35-5.23(\mathrm{~m}, 2 \mathrm{H}), 4.63-4.52(\mathrm{~m}, 3 \mathrm{H}), 4.50-4.48(\mathrm{~m}, 1 \mathrm{H}), 3.98(\mathrm{dd}, J=18 \mathrm{~Hz}$, and $J=$ $4.8 \mathrm{~Hz}, 1 \mathrm{H}), 3.89(\mathrm{dd}, J=16.8 \mathrm{~Hz}$ and $J=3.2 \mathrm{~Hz}, 1 \mathrm{H}), 3.55-3.50(\mathrm{~m}, 1 \mathrm{H}), 3.49-3.36(\mathrm{~m}, 1 \mathrm{H})$, 2.40-2.38 (m, 1H), 2.37-2.10 (m, 1H), 2.04-1.97 (m, 1H), 1.92-1.82 (m, 1H), 1.68-1.54 (m, 3H), 1.45 (s, 9H), 0.96-0.93 (m, 6H), Mass (CI method) (m/z): $427\left(\mathrm{M}^{+}+2,100\right), 371(78), 327(80)$.

Spectral data for 12. $[\alpha]_{\mathrm{D}}=-71.6(\mathrm{C}, 0.5, \mathrm{MeOH})$, IR (Neat): $3326,2930,1653 \mathrm{~cm}^{-1},{ }^{1} \mathrm{H}$ NMR $\left(400 \mathrm{MHz}, \mathrm{CDCl}_{3}\right): \delta 7.30-7.19(\mathrm{~m}, 5 \mathrm{H}), 7.11(\mathrm{~d}, \mathrm{~J}=6.5 \mathrm{~Hz}, 2 \mathrm{H}), 5.94-5.84(\mathrm{~m}, 1 \mathrm{H}), 5.35-5.24$ $(\mathrm{m}, 2 \mathrm{H}), 4.87-4.82(\mathrm{~m}, 1 \mathrm{H}), 4.64-4.62(\mathrm{~m}, 2 \mathrm{H}), 4.57-4.54(\mathrm{~m}, 1 \mathrm{H}), 3.89(\mathrm{dd}, J=17.2 \mathrm{~Hz}$ and $J=$ $4.6 \mathrm{~Hz}, 1 \mathrm{H}), 3.71(\mathrm{dd}, J=17.2 \mathrm{~Hz}$ and $J=4 \mathrm{~Hz}, 1 \mathrm{H}), 3.29-3.25(\mathrm{~m}, 2 \mathrm{H}), 3.24-3.19(\mathrm{~m}, 1 \mathrm{H})$, 3.02-2.96 (m, 1H), 2.39-2.33 (m, 1H), 1.95-1.90 (m, 2H), 1.80-1.73 (m, 1H), $1.47(\mathrm{~s}, 9 \mathrm{H})$, Mass (CI method) (m/z): $460\left(\mathrm{M}^{+}+1,22\right), 405$ (46), 361 (100).

Spectral data for 13. IR (Neat): 3313, 2977, 1742, 1655, $1523 \mathrm{~cm}^{-1},{ }^{1} \mathrm{H}$ NMR (200 MHz, $\left.\mathrm{CDCl}_{3}\right): \delta$ 7.29-7.06 (m, 6H), 5.98-5.78 (m, 1H), $5.33(\mathrm{bs}, \mathrm{NH}), 5.27-5.22(\mathrm{~m}, 2 \mathrm{H}), 4.81-4.77(\mathrm{~m}$, $1 \mathrm{H}), 4.60-4.53(\mathrm{~m}, 2 \mathrm{H}), 4.08-3.70(\mathrm{~m}, 2 \mathrm{H}), 3.60-3.25(\mathrm{~m}, 3 \mathrm{H}), 3.13-3.09(\mathrm{~m}, 2 \mathrm{H}), 2.04-2.01(\mathrm{~m}$, $1 \mathrm{H}), 1.99-.83(\mathrm{~m}, 3 \mathrm{H}), 1.46(\mathrm{~s}, 9 \mathrm{H})$, Mass (CI method) $(\mathrm{m} / \mathrm{z}): 460\left(\mathrm{M}^{+}+1,5\right), 404(33), 360$ (100).

Spectral data for 14. $[\alpha]_{\mathrm{D}}=-115.0(\mathrm{C}, 0.1, \mathrm{MeOH})$, IR (Neat): 3355, 3303, 2999, 1758, 1685 , $1646 \mathrm{~cm}^{-1},{ }^{1} \mathrm{H}$ NMR $\left(200 \mathrm{MHz}, \mathrm{CDCl}_{3}\right): 7.47$ (bs, NH), 7.26-7.19 (m, 6H), 5.91-5.80 (m, 1H), 5.36-5.21 (m, 2H), $4.61(\mathrm{~d}, J=5.8 \mathrm{~Hz}, 2 \mathrm{H}), 4.45-4.41(\mathrm{~m}, 2 \mathrm{H}), 4.22-4.03(\mathrm{~m}, 1 \mathrm{H}), 3.88-3.75(\mathrm{~m}$, $1 \mathrm{H}), 3.66-3.39(\mathrm{~m}, 2 \mathrm{H}), 2.97(\mathrm{~d}, \mathrm{~J}=7.8 \mathrm{~Hz}, 2 \mathrm{H}), 2.27-2.17(\mathrm{~m}, 1 \mathrm{H}), 1.91-1.80(\mathrm{~m}, 3 \mathrm{H}), 1.40$ (s, 9H), Mass (CI method) (m/z): $313\left(\mathrm{M}^{+}+1,7\right), 257$ (50), 213 (100).

Spectral data for 15. IR (Neat): 3293, 3085, 2975, 1732, 1664, $1635 \mathrm{~cm}^{-1},{ }^{1} \mathrm{H}$ NMR (400 MHz, $\left.\mathrm{CDCl}_{3}\right): \delta 7.20(\mathrm{bs}, \mathrm{NH}), 6.50(\mathrm{bs}, 1 \mathrm{H}), 5.95-5.77(\mathrm{~m}, 2 \mathrm{H}), 5.35-5.24(\mathrm{~m}, 2 \mathrm{H})$, 5.10-5.00 (m, 
2H), 4.64-4.60 (m, 3H), 4.16-4.00 (m, 3H), 3.60-3.56 (m, 1H), 3.48-3.41 (m, 1H), 2.43-2.41 (m, 4H), 2.21-2.18 (m, 1H), 2.15-1.90 (m, 3H), Mass (CI method) $(\mathrm{m} / \mathrm{z}): 352\left(\mathrm{M}^{+}+2,100\right)$.

Spectral data for 16. $[\alpha]_{\mathrm{D}}=-106.80(\mathrm{C}, 0.5, \mathrm{MeOH})$, IR (Neat): 3321, 3017, 2960, 1739, $1646 \mathrm{~cm}^{-1},{ }^{1} \mathrm{H}$ NMR $\left(400 \mathrm{MHz}, \mathrm{CDCl}_{3}\right): \delta 6.93(\mathrm{bs}, \mathrm{NH}), 6.4(\mathrm{bs}, \mathrm{NH}), 5.95-5.78(\mathrm{~m}, 2 \mathrm{H}), 5.35-$ $5.23(\mathrm{~m}, 2 \mathrm{H}), 5.10-5.00(\mathrm{~m}, 2 \mathrm{H}), 4.63-4.61(\mathrm{~m}, 2 \mathrm{H}), 4.59-4.51(\mathrm{~m}, 2 \mathrm{H}), 4.11(\mathrm{dd}, J=13.6 \mathrm{~Hz}$, and $J=3.6 \mathrm{~Hz}, 1 \mathrm{H}), 4.00(\mathrm{dd}, J=17.6 \mathrm{~Hz}$ and $J=4 \mathrm{~Hz}, 1 \mathrm{H}), 3.58-3.53(\mathrm{~m}, 1 \mathrm{H}), 3.46-3.39(\mathrm{~m}$, $1 \mathrm{H}), 2.43-2.30(\mathrm{~m}, 4 \mathrm{H}), 2.09-2.18(\mathrm{~m}, 1 \mathrm{H}), 2.05-1.85(\mathrm{~m}, 3 \mathrm{H}), 1.7-1.5(\mathrm{~m}, 3 \mathrm{H}), 0.98-0.96(\mathrm{~m}$, $6 \mathrm{H})$, Mass (CI method) (m/z): $409\left(\mathrm{M}^{+}+2,100\right), 337$ (40).

Spectral data for 17. $[\alpha]_{\mathrm{D}}=-73.4(\mathrm{C}, 0.5, \mathrm{MeOH})$, IR (Neat): 3264, 2074, 2933, 1759, 1671, 1636, $1554 \mathrm{~cm}^{-1},{ }^{1} \mathrm{H}$ NMR (400 MHz, $\left.\mathrm{CDCl}_{3}\right): \delta$ 7.27-7.20 (m, 4H), 7.18-7.09 (m, 2H), 6.39 (bs, $\mathrm{NH}), 5.93-5.80(\mathrm{~m}, 2 \mathrm{H}), 5.35-5.25(\mathrm{~m}, 2 \mathrm{H}), 5.12-5.01(\mathrm{~m}, 2 \mathrm{H}), 4.88-4.84(\mathrm{~m}, 1 \mathrm{H}), 4.64-4.62(\mathrm{~m}$, $2 \mathrm{H}), 4.56-4.53(\mathrm{~m}, 1 \mathrm{H}), 4.03(\mathrm{dd}, J=17.6 \mathrm{~Hz}$ and $J=4.8 \mathrm{~Hz}, 1 \mathrm{H}), 3.84(\mathrm{dd}, J=18 \mathrm{~Hz}$ and $J=$ $4 \mathrm{~Hz}, 1 \mathrm{H}), 3.33-3.30(\mathrm{~m}, 2 \mathrm{H}), 3.20(\mathrm{dd}, J=13.6 \mathrm{~Hz}$ and $J=5.6 \mathrm{~Hz}, 1 \mathrm{H}), 3.01(\mathrm{dd}, J=13.6 \mathrm{~Hz}$ and $J=7.2 \mathrm{~Hz}, 1 \mathrm{H}), 2.43-2.32(\mathrm{~m}, 4 \mathrm{H}), 1.97-1.92(\mathrm{~m}, 3 \mathrm{H}), 1.84-1.77(\mathrm{~m}, 1 \mathrm{H})$, Mass (CI method) $(\mathrm{m} / \mathrm{z}): 443\left(\mathrm{M}^{+}+2,100\right)$

Spectral data for 18. IR (Neat): 3307, 3068, 2951, 1743, 1645, $1534 \mathrm{~cm}^{-1},{ }^{1} \mathrm{H}$ NMR (200 MHz, $\left.\mathrm{CDCl}_{3}\right) \delta$ 7.26-7.23 (m, 3H), 7.12-7.08 (m, 2H), 6.95 (d, J=7.3 Hz, NH), 6.42 (bs, NH), 5.85$5.76(\mathrm{~m}, 2 \mathrm{H}), 5.30-4.96(\mathrm{~m}, 4 \mathrm{H}), 4.83-4.77(\mathrm{~m}, 1 \mathrm{H}), 4.58-4.49(\mathrm{~m}, 3 \mathrm{H}), 4.06(\mathrm{dd}, J=18 \mathrm{~Hz}$, and $J=4.40 \mathrm{~Hz}, 1 \mathrm{H}), 3.89(\mathrm{dd}, J=18 \mathrm{~Hz}$ and $J=3.9 \mathrm{~Hz}, 1 \mathrm{H}), 3.44-3.33(\mathrm{~m}, 2 \mathrm{H}), 3.12-3.08(\mathrm{~m}, 2 \mathrm{H})$, 2/35-2.33 (m, 4H), 2.29-2.28 (m, 1H), 2.21-1.80 (m, 3H), Mass (CI method) (m/z): $442\left(\mathrm{M}^{+}+1\right.$, 100).

Spectral data for 19. mp. $95-97{ }^{\circ} \mathrm{C},[\alpha]_{\mathrm{D}}=-147(\mathrm{C}, 0.1, \mathrm{MeOH}), \mathrm{IR}$ (Neat): 3282, 3075, 2929, 1757, 1640, $1554 \mathrm{~cm}^{-1},{ }^{1} \mathrm{H}$ NMR $\left(400 \mathrm{MHz}, \mathrm{CDCl}_{3}\right) \delta 7.42(\mathrm{t}, J=5.35 \mathrm{~Hz}, 1 \mathrm{H}), 7.31-7.20(\mathrm{~m}$, $5 \mathrm{H}), 6.34(\mathrm{bs}, 1 \mathrm{H}), 5.93-5.74(\mathrm{~m}, 2 \mathrm{H}), 5.33-5.21(\mathrm{~m}, 2 \mathrm{H}), 5.06-4.97(\mathrm{~m}, 2 \mathrm{H}), 4.77-4.72(\mathrm{~m}, 1 \mathrm{H})$, 4.63-4.59 (m, 2H), 4.52-4.50 (m, 1H), $4.12(\mathrm{dd}, J=18 \mathrm{~Hz}$ and $J=6.1 \mathrm{~Hz}, 1 \mathrm{H}), 3.8(\mathrm{dd}, J=$ $18 \mathrm{~Hz}$ and $J=5.1 \mathrm{~Hz}, 1 \mathrm{H}), 3.66-3.61(\mathrm{~m}, 1 \mathrm{H}), 3.07-2.97(\mathrm{~m}, 2 \mathrm{H}), 2.67-2.61(\mathrm{~m}, 1 \mathrm{H}), 2.37-2.26$ $(\mathrm{m}, 4 \mathrm{H}), 2.24-2.21(\mathrm{~m}, 1 \mathrm{H}), 1.97-1.82(\mathrm{~m}, 1 \mathrm{H}), 1.80-1.71(\mathrm{bs}, 1 \mathrm{H}), 1.70-1.55(\mathrm{~m}, 1 \mathrm{H})$, Mass (CI method) (m/z): $443\left(\mathrm{M}^{+}+2,100\right)$.

Spectral data for 16C. $[\alpha]_{\mathrm{D}}=-59.80(\mathrm{C}, 0.1, \mathrm{MeOH})$, IR (Neat): $3315,2956,1730,1648 \mathrm{~cm}^{-1}{ }^{1}$ H NMR (400 MHz, $\left.\mathrm{CDCl}_{3}\right) \delta 6.98(\mathrm{~d}, \mathrm{~J}=8.1 \mathrm{~Hz}, \mathrm{NH}), 6.36-6.34$ (bs, NH), 4.71-4.64 (m, 3H), 4.26-4.22 (m, 1H), 4.04-4.01 (m, 1H), 3.76-3.73 (m, 1H), 3.46-3.39 (m, 2H), 2.42-2.36 (m, 2H), 2.29-2.26 (m, 1H), 2.02-1.95 (m, 3H), 1.80-1.47 (m, 9H), 0.95-.90 (m, 6H), Mass (CI method) $(\mathrm{m} / \mathrm{z}): 382\left(\mathrm{M}^{+}+1,100\right)$.

Spectral data for 17C. $[\alpha]_{\mathrm{D}}=-84.80(\mathrm{C}, 0.25, \mathrm{MeOH})$, IR (Neat): $3314,2927,1729,1645 \mathrm{~cm}^{-1}$, ${ }^{1} \mathrm{H}$ NMR $\left(200 \mathrm{MHz}, \mathrm{CDCl}_{3}\right) \delta 7.39(\mathrm{~d}, J=7.2 \mathrm{~Hz}, 1 \mathrm{H}), 7.28-7.23(\mathrm{~m}, 3 \mathrm{H}), 7.07-7.02(\mathrm{~m}, 2 \mathrm{H})$, 6.24-6.23 (m, 1H), 4.90-4.85 (m, 1H), $4.63(\mathrm{~d}, J=7.3 \mathrm{~Hz}, 1 \mathrm{H}), .446-4.40(\mathrm{~m}, 1 \mathrm{H}), 4.29-4.23(\mathrm{~m}$, $1 \mathrm{H}), 3.99-3.94(\mathrm{~m}, 1 \mathrm{H}), 3.60-3.55(\mathrm{~m}, 1 \mathrm{H}), 3.47-3.29(\mathrm{~m}, 4 \mathrm{H}), 3.08-3.03(\mathrm{~m}, 1 \mathrm{H}), 2.38-2.30(\mathrm{~m}$, $1 \mathrm{H}), 2.29-2.24(\mathrm{~m}, 2 \mathrm{H}), 1.94-1.80(\mathrm{~m}, 2 \mathrm{H}), 1.79-1.60(\mathrm{~m}, 4 \mathrm{H}), .58-1.44(\mathrm{~m}, 1 \mathrm{H}), 1.15-1.05(\mathrm{~m}$, $1 \mathrm{H})$, Mass (CI method) (m/z): $416\left(\mathrm{M}^{+}+1,100\right)$. 
Spectral data for 19C. $[\alpha]_{D}=-105.33(\mathrm{C}, 0.375, \mathrm{MeOH})$, IR (Neat): 3395, 2931, 1727, $1637 \mathrm{~cm}^{-1},{ }^{1} \mathrm{H}$ NMR $\left(400 \mathrm{MHz}, \mathrm{CDCl}_{3}\right) \delta 7.58(\mathrm{bs}, 1 \mathrm{H}), 7.32-7.24(\mathrm{~m}, 3 \mathrm{H}), 7.21-7.19(\mathrm{~m}, 2 \mathrm{H})$, $6.18(\mathrm{~d}, \mathrm{~J}=7.6 \mathrm{~Hz}, 1 \mathrm{H}), 4.92-4.86(\mathrm{~m}, 1 \mathrm{H}), 4.47$ (d, $J=7.8 \mathrm{~Hz}, 1 \mathrm{H}), 4.32-4.27(\mathrm{~m}, 1 \mathrm{H}), 4.10-$ $4.04(\mathrm{~m}, 4 \mathrm{H}), 3.63-3.59(\mathrm{~m}, 1 \mathrm{H}), 3.05-2.96(\mathrm{~m}, 2 \mathrm{H}), 2.64-2.57(\mathrm{~m}, 1 \mathrm{H}), 2.36-2.30$ (m, 3H), 2.19$2.12(\mathrm{~m}, 2 \mathrm{H}), 1.80-1.68(\mathrm{~m}, 2 \mathrm{H}), 1.58-1.51(\mathrm{~m}, 2 \mathrm{H}), 1.49-1.40(\mathrm{~m}, 2 \mathrm{H})$, Mass (CI method) $(\mathrm{m} / \mathrm{z})$ : $416\left(\mathrm{M}^{+}+1,100\right)$.

\section{Acknowledgements}

BMR thanks Dr. Reddy's Research Foundation and Dr. Reddy's Laboratories Ltd for the fellowship and Infrastructure facilities. BMR also thanks analytical department for spectral data.

\section{Reference}

1. Fix, J. A., Pharm. Res. 1996, 13, 1760.

2. Selected reviews on $\beta$-turn mimics: (a) Rose, G. D.; Gierasch, L. M.; Smith, J. A. In Advances in Protein Chemistry; Anfinsen, C. B., Edsall, J. T., Richards, F. M., Eds.; Academic: Orlando, FL, 1985; Vol. 37, pp 1-109. (b) Giannis, A.; Kolter, T. Angew. Chem., Int. Ed. 1993, 32, 1244. (c) Nagai, U.; Sato, K.; Nakamura, R.; Kato, R. Tetrahedron 1993, 49, 3577. (d) Olson, G. L.; Bolin, D. R.; Bonner, M. P.; Bos, M.; Cook, C. M.; Fry, D. C.; Graves, B. J.; Hatada, M.; Hill, D. E.; Kahn, M.; Madison, V. S.; Rusiecki, V. K.; Sarabu, R.; Sepinwall, J.; Vincent, G. P.; Voss, M. E. J. Med. Chem. 1993, 36, 3039. (e) Mueller, G. Angew. Chem., Int. Ed. 1997, 35, 2767. (f) MacDonald, M.; Aube, J. Curr. Org. Chem. 2001, 5, 417. (g) Hanessian, S.; McNaughton-Smith, G.; Lombart, H.-G.; Lubell, W. D. Tetrahedron 1997, 53, 12789. (h) Burgess, K. Acc. Chem. Res. 2001, 34, 826. (i) Nubbemeyer, U. Top. Curr. Chem. 2001, 216, 125. (j) Souers, A. J.; Ellman, J. A. Tetrahedron 2001, 57, 7431. (k) Eguchi, M.; Kahn, M. Mini-Rev. Med. Chem. 2002, 2, 447. (1) Hruby, V. J. Nat. Rev. Drug Discovery 2002, 1, 847. (m) Suat Kee, K.; Jois, S. D. S. Curr. Pharm. Des. 2003, 9, 1209. (n) Ahn, J.-M.; Boyle, N. A.; MacDonald, M. T.; Janda, K. D. Mini-Rev. Med. Chem. 2002, 2, 463.

3. Selected recent papers on $\beta$-turn mimics: (a) Zhang, J.; Xiong, C.; Ying, J.; Wang, W.; Hruby, V. J. Org. Lett. 2003, 5, 3115. (b) Lee, H. B.; Pattarawarapan, M.; Roy, S.; Burgess, K. Chem. Commun. 2003, 1674. (c) Xiong, C.; Zhang, J.; Davis, P.; Wang, W.; Ying, J.; Porreca, F.; Hruby, V. J. Chem. Commun. 2003, 1598. (d) Baek, B.; Lee, M.; Kim, K.; Cho, U.; Boo, D. W.; Shin, I. Org. Lett. 2003, 5, 971. (e) Boruah, A.; Rao, I. N.; Nandy, J. P.; Kumar, S. K.; Kunwar, A. C.; Iqbal, J. J. Org. Chem. 2003, 68, 5006. (f) Luppi, G.; Lanci, D.; Trigari, V.; Garavelli, M.; Garelli, A.; Tomasini, C. J. Org. Chem. 2003, 68, 1982. (g) 
Kaul, R.; Deechongkit, S.; Kelly, J. W. J. Am. Chem. Soc. 2002, 124, 11900. (h) Han, Y.; Giragossian, C.; Mierke, D. F.; Chorev, M. J. Org. Chem. 2002, 67, 5085. (i) Wels, B.; Kruijtzer, J. A. W.; Liskamp, R. M. J. Org. Lett. 2002, 4, 2173. (j) Grieco, P.; Campiglia, P.; Gomez-Monterrey, I.; Novellino, E. Tetrahedron Lett. 2002, 43, 1197. (k) Gibbs, A. C.; Bjorndahl, T. C.; Hodges, R. S.; Wishart, D. S. J. Am. Chem. Soc. 2002, 124, 1203. (1) Hoffmann, T.; Lanig, H.; Waibel, R.; Gmeiner, P. Angew. Chem., Int. Ed. 2001, 40, 3361. (m) Sukopp, M.; Marinelli, L.; Heller, M.; Brandl, T.; Goodman, S. L.; Hoffmann, R. W.; Kessler, H. Helv. Chim. Acta 2002, 85, 4442. (n) Halab, L.; Lubell, W. D. J. Am. Chem. Soc. 2002, 124, 2474.

4. Banerji, B.; Bhattacharya, M.; Madhu, R. B.; Das, S. K.; Iqbal, J. Tetrahedron Lett. 2002 , 43, 6473. 